\title{
FACTORES PERINATALES ASOCIADOS A SUPERVIVENCIA DE RECIÉN NACIDOS CON EXTREMADAMENTE BAJO PESO AL NACER.INSTITUTO NACIONAL MATERNO PERINATAL-2010
}

\author{
Rossana Cecilia Andrade Chávez ${ }^{1}$
}

\begin{abstract}
RESUMEN
Objetivo. Determinar los factores asociados a supervivencia de recién nacidos de extremadamente bajo peso al nacer (RNEBPN) en el Instituto Nacional Materno Perinatal (INMP). Métodos. Estudio de casos y controles en la Unidad de Cuidados Intensivos Neonatales (UCIN) del INMP. Se revisaron las historias clínicas de todos los RNEBPN, nacidos en el INMP entre enero y diciembre de 2010, los cuales fueron divididos en dos grupos: grupo 1 (casos), RNEBPN vivos al alta y; grupo 2 (controles), RNEBPN fallecidos al alta. En ambos grupos se buscó los factores a los cuales estuvieron expuestos. Se realizó análisis bivariado, calculó del Odd radio con intervalo de confianza al $95 \%$ y regresión logística. Resultados. Durante el periodo de estudio ingresaron a la UCIN 85 RNEBPN; 41 fueron dados de alta vivos (casos) con una supervivencia global de $48 \%$. Al comparar las características entre grupos, se encontró diferencia significativa $(p<0,05)$ en peso de nacimiento y edad gestacional. Los factores con asociación significativa mediante regresión logística para supervivencia fueron: nacimiento por cesárea (OR 14,1; IC 2,9-67,9), sexo femenino (OR 10,1; IC 2,4-42,2), edad gestacional $\geq$ a 28 semanas (OR 5,3; IC 1,2-23,1) y uso de indometacina (OR 5,34; IC 1,3-21,8). Conclusiones. La supervivencia de RNEBPN en el INMP fue de $48 \%$. El nacimiento por cesárea, el sexo femenino y la edad gestacional $\geq a$ 28 semanas fueron factores importantes para la supervivencia.
\end{abstract}

Palabras clave: Supervivencia neonatal; Recién nacido de extremo bajo peso(fuente: DeCS BIREME).

\section{PERINATAL FACTORS ASSOCIATED WITH NEWBORN SURVIVAL OF EXTREMELY LOW BIRTH WEIGHT}

\begin{abstract}
Objective. To determine factors associated with mortality in ELBW infants in the Maternal Perinatal National Institute (INMP). Methods. A case-control study in the NICU of the INMP. We reviewed the medical records of all the ELBW infants, born in INMP since January to December of 2010, which were divided into two groups: group 1 (cases), ELBW infants alive at discharge; group 2 (controls), ELBW infants dead at discharge; both groups sought the factors to which they were exposed. We performed bivariate analysis, calculation from the odd radio with confidence interval of $95 \%$ and logistic regression. Results. During the study period were admitted to the NICU 85 ELBW infant, 41 were discharge alive (cases) with a survival rate of $48 \%$. Comparing the characteristics between groups there was significant difference $(p<0.05)$ in birth weight and gestational age. The factors with significantly association through logistic regression for survival, were: Caesarean section birth (OR 14,07; Cl 2,9-67,9), famele (OR 10,12; Cl 2,43-42,18), gestational age $\geq$ a 28 weeks (OR 5,3; Cl 1,21-23,11) and using indomethacin (OR 5,34; $\mathrm{Cl} 1,31-21,80)$. Conclusions. The survival of the ELBW infant in the INMP was $48 \%$. The caesarean birth, the female gender and the gestational age $\geq 28$ weeks were significant factors for survival.
\end{abstract}

Keywords: Neonatal survival; Extremely low birth weight infants(source: MeSH NLM).

\section{INTRODUCCION}

La sobrevida de los recién nacidos de extremadamente bajo peso al nacer (RNEBPN) ha experimentado una mejoría significativa en las últimas décadas, ello debido a los avances de la medicina perinatal. Este se ve reflejado en el descenso de las tasas de mortalidad perinatal y neonatal, cuyo impacto se traduce asimismo en la reducción de la mortalidad infantil ${ }^{1}$.

Existen numerosas publicaciones en la literatura médica acerca de la sobrevida y pronóstico de los recién nacidos prematuros extremos, sin embargo es difícil establecer comparaciones entre ellas, ya que la mayoría difieren en el tamaño de la muestra, en la heterogeneidad de las poblaciones estudiadas y en el uso de diferentes definiciones.

Los recién nacidos con extremadamente bajo peso al nacer, son aquellos que, independiente de su edad gestacional, tienen un peso de nacimiento menor a 1000 gramos. Es un término acuñado en la década de 1990 en vista de la creciente cantidad de recién nacidos con peso menor a 1000 gramos que sobrevivían ${ }^{2}$.

Médico Neonatóloga. Departamento de Neonatología. Instituto Nacional Materno Perinatal

Recibido: 10-10-13 Aprobado: 3-12-13 
La incidencia de RNEBPN varía de centro a centro y va de 0,33 a $0,7 \%$. En Quebec entre 1981 y 1993 los RN con peso entre 500 a 999 gramos represento el 0,33\% ${ }^{2}$. En el Instituto Nacional Materno Perinatal (INMP), donde se llevó cabo este trabajo; en el 2010 se produjeron 16878 partos, de ellos los el $0,7 \%$ fueron RBEBPN ${ }^{3}$.

Los factores con un valor predictivo importante para la supervivencia de los RBEBPN son edad gestacional más avanzada, mayor peso al nacer, sexo femenino, neonato único y ausencia de retardo de crecimiento intrauterino ${ }^{2}$. En los rangos más bajos de edad gestacional (EG) y peso de nacimiento (PN) ocurren cambios significativos en la mortalidad por cada semana de gestación adicional y por cada 100 gramos que incremente el peso, por ello resulta útil y necesario desglosar las tablas de sobrevida por semana de $E G$ y cada 100 gramos ${ }^{4}$.

El presente estudio tuvo como objetivo determinar los factores perinatales asociados a la supervivencia de recién nacidos con extremadamente bajo peso al nacer, atendidos en el Instituto Nacional Materno Perinatal. Dicho conocimiento contribuirá a mejor el manejo de este grupo de recién nacidos y a disminuir la mortalidad neonatal, componente importante de la mortalidad infantil

\section{MATERIAL Y MÉTODOS}

Se realizó un estudio de casos y controles. La población del estudio se dividió en 2 grupos: los casos conformado por todos los recién nacidos de extremadamente bajo peso al nacer RNEBPN, nacidos en el Instituto Nacional Materno Perinatal INMP durante el año 2010 y vivos al alta, y los controles conformado por todos los RNEBPN, nacidos en el INMP durante el año 2010 y fallecidos al alta En ambos grupos y retrospectivamente, se buscó los factores perinatales a los cuales estuvieron expuestos.

La población seleccionada fue la totalidad de RNEBPN, nacidos en el INMP de enero a diciembre de 2010.

Se incluyeron como casos todos los recién nacidos menores de 1000 gr y como controles los menores de 1000 gr fallecidos al alta durante el periodo de investigación. Se excluyeron los recién nacidos menores a $1000 \mathrm{gr}$ con malformaciones congénitas, con información incompleta 0 nacidos en otras instituciones

Se evaluó la supervivencia del recién nacido de extremadamente bajo peso al nacer y su relación con los factores prenatales (control prenatal, pre-eclampsia, embarazo múltiple, ruptura prematura de membranas, corioamnionitis y administración de corticoides prenatales), natales (tipo de parto, reanimación cardiopulmonar, depresión severa, peso al nacer, edad gestacional y sexo) y post natales (enfermedad de membrana hialina, sepsis tardía, enterocolitis necrotizante, shock séptico, hemorragia intraventricular, persistencia del conducto arterioso, hipernatremia, hiperglicemia, ventilación mecánica asistida, uso de surfactante, cateterismo de vasos umbilicales, uso de indometacina, uso de inotrópicos y uso de insulina).

Se revisó el libro de ingresos de la UCIN, de él se extrajo la relación de RNEBPN que ingresaron a la UCIN durante el periodo de estudio. En archivo central, se solicitó las historias clínicas maternas y neonatales correspondientes, con las cuales se llenó del instrumento recolección y de la base de datos hecha en el programa estadístico SPSS versión 21.

Se usó el programa estadístico SPSS versión 21. Estadística descriptiva fue usada en el análisis preliminar de datos de ambos grupos. Se realizó análisis bivariado y se aplicó el test de Chi cuadrado para hallar asociación, considerándose significativa cuando el valor de $p<$ de 0.05. En aquellos factores que se encontró asociación significativa, se calculó el Odds ratio (OR) con un intervalo de confianza al 95\%. Posteriormente se realizó regresión logística para el cálculo del peso de cada factor.

El proyecto fue aprobado por la oficina de investigación del Instituto Nacional Materno Perinatal y conto con el permiso del jefe de servicio.

\section{RESULTADOS}

Durante el periodo de estudio ingresaron a la UCIN del Instituto Nacional Materno Perinatal 96 recién nacidos de extremadamente bajo peso, no se ubicó las historias clínicas en 10 y uno fue transferido a EsSalud. Entraron al estudio 85 RNEBPN (el 88,5\%); 41 fueron dados de alta vivos (grupo casos) y 44 fallecieron (grupo control). El porcentaje global de supervivencia fue de 48,2\% (41/85). La clasificación según edad gestacional y peso de nacimiento, de acuerdo a la condición de alta, se observa en las tablas 1 y 2 .

Para comparar las medias del peso de nacimiento y la edad gestacional entre grupo de estudio y grupo control, se usó la t de Student (ambas variables tuvieron distribución normal), los resultados los podemos ver en la tabla 3.

Los valores del test de Apgar al minuto y a los 5 minutos, no tuvieron distribución normal y para comprar los grupos (supervivientes y fallecidos) se usó un test no paramétrico

Tabla 1. RN EBPN según edad gestacional y condición de alta. INMP-2010

\begin{tabular}{lcccccc}
\hline \multirow{2}{*}{ Edad gestacional } & \multicolumn{2}{c}{ Vivos } & \multicolumn{2}{c}{ Fallecidos } & \multicolumn{2}{c}{ Total } \\
\cline { 2 - 7 } & No & $\%$ & No & $\%$ & No & $\%$ \\
\hline Menor de 26 sem & 1 & 6,7 & 14 & 93,3 & 15 & 100,0 \\
\hline 26 a 27 sem & 12 & 41,4 & 17 & 58,6 & 29 & 100,0 \\
28 a 29 sem & 11 & 64,7 & 6 & 35,3 & 17 & 100,0 \\
30 a 31 sem & 11 & 73,7 & 4 & 26,7 & 15 & 100,0 \\
32 a 33 sem & 5 & 71,4 & 2 & 28,6 & 7 & 100,0 \\
Mayor de 33 sem & 1 & 50,0 & 1 & 50,0 & 2 & 100,0 \\
Total & 41 & 48,2 & 44 & 51,8 & 85 & 100,0 \\
\hline
\end{tabular}


Tabla 2. RN EBPN según Peso al nacer y condición de alta. INMP-2010

\begin{tabular}{lcccccc}
\hline \multirow{2}{*}{ Peso Nacimiento } & \multicolumn{2}{c}{ Vivo } & \multicolumn{3}{c}{ Fallecidos } & \multicolumn{2}{c}{ TOTAL } \\
\cline { 2 - 7 } & No & $\%$ & No & $\%$ & No & $\%$ \\
\hline Menor de $500 \mathrm{~g}$ & 0 & 0,0 & 2 & 100,0 & 2 & 100,0 \\
500 a $599 \mathrm{~g}$ & 0 & 0,0 & 6 & 100,0 & 6 & 100,0 \\
600 A $699 \mathrm{~g}$ & 5 & 45,5 & 6 & 54,5 & 11 & 100,0 \\
700 a $799 \mathrm{~g}$ & 11 & 44,0 & 14 & 56,0 & 25 & 100,0 \\
800 a $899 \mathrm{~g}$ & 7 & 46,7 & 8 & 53,3 & 15 & 100,0 \\
900 a $999 \mathrm{~g}$ & 18 & 69,2 & 8 & 30,8 & 26 & 100,0 \\
TOTAL & 41 & 48,2 & 44 & 51,8 & 85 & 100,00 \\
\hline
\end{tabular}

Tabla 3. Comparación del PN y la EG en RN EBPN. INMP-2010

\begin{tabular}{lccccc}
\hline \multirow{2}{*}{ Característica } & \multicolumn{2}{c}{ Vivos } & \multicolumn{2}{c}{ Fallecidos } & $\mathbf{p}$ \\
\cline { 2 - 6 } & $\mathbf{X}$ & $\mathbf{D E}$ & $\mathbf{X}$ & $\mathbf{D E}$ & \\
\hline Edad Gestacional (sem) & 28,9 & 2,1 & 27 & 2,4 & $0,001^{*}$ \\
Peso de Nacimiento $(\mathrm{g})$ & 846 & 115 & 755 & 134 & $0,001^{*}$ \\
\hline X: media & & & & & \\
DE: desviación estándar \\
*t de Student
\end{tabular}

(la U de Mann Whitney); los supervivientes tuvieron valores más altos, tanto al minuto como a los 5 minutos respecto al grupo control, pero no hubo diferencias significativas ( $p$ $>0,05)$.

El análisis bivariado para los factores prenatales, natales y postnatales los vemos en la tabla 4

Para factores prenatales, natales y postnatales con diferencia significativa entre grupos $(p<0,05)$, se calculó el Odds Ratio (OR) con un intervalo de confianza de $95 \%$, los resultados se observan en la tabla 5 . Los factores con

Tabla 4. Análisis bivariado entre factores prenatales, natales, postnatales y condición de alta. INMP-2010

\begin{tabular}{|c|c|c|c|c|c|}
\hline \multirow[t]{2}{*}{ Característica } & \multirow[b]{2}{*}{ No } & \multirow[b]{2}{*}{$\%$} & \multicolumn{2}{|c|}{ Fallecidos } & \multirow[t]{2}{*}{$\mathbf{p}$} \\
\hline & & & No & $\%$ & \\
\hline \multicolumn{6}{|l|}{ FACTORES PRENATALES } \\
\hline Con CPN & 7 & 17,1 & 5 & 11,4 & 0,45 \\
\hline Pre eclampsia & 13 & 31,7 & 9 & 20,5 & 0,23 \\
\hline Embarazo múltiple & 3 & 7,3 & 8 & 18,2 & 0,14 \\
\hline RPM & 10 & 24,6 & 4 & 9,1 & 0,05 \\
\hline Corioamnionitis & 7 & 17,1 & 4 & 9,1 & 0,27 \\
\hline Corticoides prenatales & 16 & 39,0 & 11 & 25,0 & 0.16 \\
\hline \multicolumn{6}{|l|}{ FACTORES NATALES } \\
\hline Cesárea & 37 & 90,2 & 20 & 45,5 & 0,0001 \\
\hline $\mathrm{RCP}$ & 15 & 36,6 & 27 & 61,4 & 0,02 \\
\hline Sexo Femenino & 27 & 65,9 & 10 & 22,7 & 0,0001 \\
\hline Peso $\geq 700 \mathrm{~g}$ & 36 & 87,8 & 30 & 68,2 & 0,03 \\
\hline Edad $\geq 28$ semanas & 28 & 68,3 & 13 & 29,5 & 0.0001 \\
\hline \multicolumn{6}{|l|}{ FACTORES POSTNATALES } \\
\hline Membrana Hialina & 36 & 87,8 & 40 & 90,9 & 0,64 \\
\hline Sepsis tardía & 24 & 58,5 & 10 & 36,8 & 0,09 \\
\hline Enterocolitis necrotizante & 0 & 0,0 & 1 & 2,3 & 0,51 \\
\hline Shock séptico & 4 & 9,8 & 7 & 15,9 & 0,39 \\
\hline Hemorragia intraventricular & 9 & 22,9 & 12 & 27,3 & 0,57 \\
\hline Conducto arterioso & 33 & 83,5 & 29 & 65,9 & 0,13 \\
\hline Ventilación mecánica & 34 & 82,9 & 42 & 95,5 & 0,06 \\
\hline Uso de surfactante & 34 & 82,9 & 39 & 88,6 & 0,45 \\
\hline Uso de indometacina & 31 & 75,6 & 22 & 50,0 & 0,01 \\
\hline Uso de inotrópicos & 6 & 14,6 & 12 & 27,3 & 0,15 \\
\hline Cateterismo umbilical & 33 & 80,5 & 37 & 84,1 & 0,66 \\
\hline Uso de insulina & 2 & 4,9 & 10 & 22,7 & 0,01 \\
\hline
\end{tabular}

Tabla 5. Factores perinatales y OR para Supervivencia. INMP-2010

\begin{tabular}{lcc}
\hline Factores perinatales & OR & IC 95\% \\
\hline RMP & 3,2 & $0,9-11,2$ \\
Cesárea & 11 & $3,4-36,4$ \\
RCP & 0,36 & $0,15-0,87$ \\
Sexo Femenino & 6,5 & $2,5-17,1$ \\
Peso $\geq$ a 700 g & 3,3 & $1,1-10,4$ \\
EG $\geq$ a 28 sem & 5,1 & $2,1-12,9$ \\
Uso de indometacina & 3,1 & $1,2-7,8$ \\
Uso de insulina & 0,17 & $0,03-0,85$ \\
\hline
\end{tabular}

OR: Odds ratio

IC: Intervalo de confianza

Tabla 6. Factores perinatales y OR corregido (regresión logística) para Supervivencia. INMP-2010

\begin{tabular}{lcc}
\hline Factores perinatales & OR & IC 95\% \\
\hline CesáreaT & 14,07 & $2,9-67,9$ \\
RCP & 0,47 & $0,12-1,8$ \\
Sexo Femenino & 10,12 & $2,43-42,2$ \\
PN $\geq$ a $700 \mathrm{~g}$ & 2,19 & $0,38-12,6$ \\
EG $\geq$ a 28 sem & 5,30 & $1,21-23,1$ \\
Uso de indometacina & 5,34 & $1,31-21,8$ \\
Uso de insulina & 0,06 & $0,04-1,2$ \\
\hline
\end{tabular}

un intervalo de confianza que no tocaba el 1 , entraron a un modelo de regresión logística (tabla 6).

\section{DISCUSIÓN}

Los embarazos de alto riesgo ha incrementado en los últimos años; ello lleva consigo el aumento de los nacimientos de recién nacidos pretérmino de muy bajo peso al nacer (menor de $1500 \mathrm{~g}$ ) y extremadamente bajo peso al nacer (menor de $1000 \mathrm{~g}$ ). Estos recién nacidos son un grupo de alto riesgo y; gracias al avance tecnológico, su sobrevida esta en incremento. Sin embargo a pesar de todos los esfuerzos y pese a la utilización de tecnología y recursos terapéuticos la mortalidad es aun alta ${ }^{5}$.

Reportamos los resultados de 85 recién nacidos de extremadamente bajo peso al nacer. La supervivencia global encontrada fue de $48 \%$; esta cifra varió de 0 a $70 \%$ según peso de nacimiento y edad gestacional (tabla 1 y 2 ). Según el peso nacer no hubo supervivencia en menores de 600 gramos, pero esta incrementa a cerca de $70 \%$ entre 900 y 1000 gramos. Según edad gestacional, en menores de 26 semanas encontramos solo $7 \%$ de supervivencia y esta incrementa a $74 \%$ a las 30 a 31 semanas.

Estos hallazgos podrían servir para fijar nuestro límite de viabilidad en prematuros nacidos en el Instituto Nacional Materno Perinatal, el cual podría ser fijado en 600 gramos y 26 semanas en las cuales la supervivencia es cercana al $40 \%$.

La supervivencia global que reportamos es superior a la reportada por Lohmann en el Hospital Cayetano Heredia, quien en el 2006 reporta una sobrevivencia de $25 \%$ para recién nacidos de extremadamente bajo peso al nacer ${ }^{6}$. Esta 
diferencia encuentra explicación en la diferente capacidad resolutiva debido al grado de equipamiento y número de personal capacitado que en ellos laboran ${ }^{7}$.

Al comparar nuestros resultados según peso (tabla 2) con el grupo NEOCOSUR, tenemos una mejor supervivencia en algunos grupos, así; para RN entre 500 y 600 gramos reportamos $0 \%$ de sobrevida versus $13 \%$; entre 600 a 699 gramos $46 \%$ versus $33 \%$; entre 700 a 799 gramos $44 \%$ versus $50 \%$. Pero al comparar nuestra supervivencia según edad gestacional, esta es mucho menor ya que NEOCOSUR reporta una supervivencia de $50 \%$ en edades gestacionales de 25 a 26 semanas y nosotros solo $7 \%$ y llegamos al $50 \%$ entre las 27 a 29 semanas ${ }^{5}$.

Si bien es cierto que la sobrevivencia es mayor que algunos hospitales del país y la región, es mucho menos que reportes del extranjero. Lemons, en el 2001, reporta una supervivencia global de $84 \%$, que como se ve es una cifra muy superior a la nuestra ${ }^{8}$. Otra vez, creemos que el grado de implementación de las UCIN y la capacitación de los profesionales que trabajan en estas explican estas diferencias tan amplias ${ }^{7}$. Este hecho también fue demostrado por Rautava et/al en el 2007, quien reporta los efectos del nacimiento en un centro nivel III versus un nivel II, con mayor mortalidad en los hospitales nivel II, incluso durante el primer año de vida ${ }^{9}$.

En lo que respecta a los factores perinatales asociados a supervivencia en los recién nacidos de extremadamente bajo peso. Se determinó la importancia del peso al nacer y la edad gestacional; así lo demuestra la tabla 3 en la cual observamos que, tanto el peso de nacimiento como la edad gestacional fueron mayores en los recién nacidos vivos al alta y tuvieron diferencia significativa $(p<0,05)$. No se encontró diferencias significativas en la valoración del Apgar ni al minuto y a los 5 minutos.

La importancia del peso y la edad gestacional en la supervivencia de los recién de extremadamente bajo peso ha sido reportada en varios trabajos, de ellos se desprende que la supervivencia es directamente proporcional al peso a ya la edad gestacional (a mayor peso y edad gestacional mayor supervivencia). Este hecho lo podemos evidenciar en las tablas 1 y 2 que muestran la supervivencia por grupos de edad gestacional y peso de nacimiento respectivamente. Este mismo hecho (aunque con cifras diferentes) fue evidenciado por otros autores; así, Lemons en 4438 recién nacidos de muy bajo peso (entre 501 y 1500 g) encontró que, entre 501 y 750 gramos el $54 \%$ sobrevivieron al alta, y sube a $86 \%$ en pesos entre 701 a 1000 gramos $^{8}$.

La supervivencia en relación al peso de nacimiento y edad gestacional encuentra explicación en el grado de madurez de órganos y sistemas alcanzados. A mayor edad gestacional, el peso de nacimiento y la madurez alcanzada serán mayores y también probabilidades supervivencia.

Después de realizar el análisis bivariado y el análisis multivariado, los factores asociados a supervivencia fueron nacimiento por cesárea, sexo femenino, la edad gestacional mayor o igual a 28 semanas y uso de indometacina. El resto de factores analizados, si bien tuvieron diferencia significativa y un OR importante, en la logística, no salieron relevantes.

El efecto del sexo femenino sobre los resultados perinatales ha sido reportado y estudiado con frecuencia. Así, Lohmann reporto que entre los recién nacidos de extremadamente bajo peso los fallecidos, predominaron los del sexo masculino. En lo que respecta al nacimiento por cesárea, los reportes son más controversiales pero con una cierta tendencia sobre los efectos positivos de esta. Caro et/al, en el Hospital de Puerto Montt, encontró que el nacimiento por cesárea se asoció a una reducción significativa del riesgo de depresión neonatal severa y muerte neonatal ${ }^{10}$.

Los efectos de la indometacina sobre la supervivencia, encuentra explicación en su efecto sobre el cierre del ductus arterioso, así se reportan que, altas dosis de indometacina resultó en una tasa global de cierre de $98,5 \%{ }^{11}$; por lo tanto, su uso evitaría los efectos deletéreos del ductus sobre la salud de este grupo de recién nacidos.

\section{REFERENCIAS BIBLIOGRÁFICAS}

1. Encuesta Demográfica y de Salud Familiar - ENDES 2011. INEI: Pág. 161.

2. Papageorgiou $A$, Bardin C. El neonato con extremadamente bajo peso. En: Neonatología, fisiopatología y manejo del recién nacido de Avery. Quinta edición. Editorial Panamericana, Madrid 2001. Pag446-74.

3. Estadísticas del INMP. Disponible en: http://inmp.gob.pe/.

4. Thórarinsdóttir B, Georgsdóttir I, Jóhannsson J, Dagbjartsson A. Extremely low birthweight infants in Iceland 1991-95. Risk factors for perinatal and neonatal death. (2009). Laeknabladid. 2009 Feb;95(2):107-11.

5. Grupo Colaborativo Neocosur. Very Low Birth Weigth infants outcome in Southamerican NICU. J. Perinatol; 2002: 202-7.

6. Lohmann P, Rodríguez M, Webb V, Rospigliosi M. Mortalidad en recién nacidos de extremo bajo peso al nacer en la unidad de neonatología del Hospital Nacional Cayetano Heredia entre enero 2000 y diciembre 2004. Rev Med Hered 2006;17:141-147.

7. Grandi C, Gonzales A, Meritano J. Riesgo de morbimortalidad neonatal de recién nacidos < de 1500 gramos asociado al volumen de pacientes, personal médico y de enfermería: una investigación multicéntrico latinoamericana. Rev Hosp Mat Inf Ramón Sardá 2010, 29(1):32-40.

8. Lemons J, Bauer C, Oh W, Korones Sh, Ann L, Et/al. (2001) Very Low Birth Weight Outcomes of the National Institute of Child Health and Human Development Neonatal Research Network, January 1995 Through December 1996. Pediatrics 2001;107, e1.

9. Rautava L, Lehtonen L, Peltola M, Korvenranta E, Korvenranta $\mathrm{H}$, et/al. The Effect of Birth in Secondary- or Tertiary-Level Hospitals in Finland on Mortality in Very Preterm Infants: A Birth-Register Study. Pediatrics 2007;119;e257.

10. Caro J, Flores G, Ortiz E, Anwandter C, Rodríguez D. Pronóstico neonatal del recién nacido de muy bajo peso: hospital regional de Puerto Montt, 2000-2005. Rev Chil Obstet Ginecol 2007; 72(5)

11. Sperandio M, Beedgen B, Feneberg R, Huppertz C, Brussau J, et/al. Effectiveness and Side Effects of an Escalating, Stepwise Approach to Indomethacin Treatment for Symptomatic Patent Ductus Arteriosus in Premature Infants Below 33 Weeks of Gestation. Pediatrics 2005;116:1361-1366. 